\title{
El problema de la obligación políitca y el principio de fair play: una evaluación crítica
}

\author{
Recibido: 3 de septiembre de 2020 • Aprobado: 7 de octubre de 2020 \\ https://doi.org/10.22395/ojum.v20n42al 1
}

Eduardo Esteban Magoja

Universidad de Buenos Aires (UBA), Buenos Aires, Argentina

eduardomagoja@derecho.uba.ar

https://orcid.org/0000-0003-3182-5219

\section{RESUMEN}

Existen distintas teorías que buscan dar una respuesta satisfactoria a la pregunta acerca de por qué las personas deben obedecer el derecho. Una de estas propuestas es el principio de fairplay (o fairness), el cual establece que si un individuo que forma parte de un esquema social de cooperación se beneficia del esfuerzo de los demás participantes tiene el deber de soportar las cargas, pues de lo contrario cometería una injusticia. Varios autores han reconocido el valor del principio, pero otros han demostrado con buenos argumentos que tiene muchas dificultades para justificar un deber de obediencia general en esquemas complejos como el Estado. El objetivo principal de este trabajo es evaluar, mediante una metodología caracterizada por la revisión de la literatura especializada, las virtudes y debilidades del principio de fairplay como teoría de la obligación política. La conclusión obtenida es que esta teoría podría ser una propuesta adecuada siempre y cuando se satisfagan determinados requisitos.

Palabras clave: fair play; fairness; obligación política, esquema cooperativo; Estado. 


\title{
The Problem of Political Obligation and the Principle of Fair Play: A Critical Assessment
}

\begin{abstract}
There are different theories that try to give a satisfactory answer to the question of why people should obey the law. One of these proposals is the principle of fair play (or fairness). It says that if a person, who is part of a social cooperation scheme, benefits from the efforts of the other participants he/she is bound to do his part; otherwise, he/she acts unfairly. Several scholars have recognized the value of the principle, but others have demonstrated with good arguments that it has many difficulties to justify a general duty of obedience in complex schemes such as the State. The main objective of the paper is to assess, by using the literature review method, the virtues and weaknesses of the principle of fair play as a theory of political obligation. The obtained conclusion is that this theory could be an appropriate proposal as long as it satisfies some requirements.
\end{abstract}

Keywords: fair play; fairness; political obligation; cooperative scheme; State 


\section{INTRODUCCIÓN}

Este trabajo es uno de los resultados de investigación desarrollado en el marco del proyecto de investigación El problema de la (des)obediencia al derecho: de la antigüedad a la actualidad" (2020-2021), acreditado y financiado por la Facultad de Derecho de la Universidad de Palermo, Argentina.

No es extraño pensar la comunidad como una empresa cooperativa que establece las condiciones mínimas para que cada uno de los integrantes pueda llevar a cabo su plan de vida y, además, mediante un trabajo conjunto y organizado, se aspire a la realización del bienestar general. Tampoco es extraño decir, en cuanto a la dinámica de su funcionamiento, que los participantes tienen una amplia gama de libertades y, al mismo tiempo, de deberes hacia los demás miembros del grupo que nacen como consecuencia de los beneficios obtenidos. En efecto, a lo largo de la historia diversos pensadores ofrecieron una explicación de este tipo en lo que respecta a la estructura de la vida en sociedad, sus funcionamiento y fines. En el Critón de Platón (2010, 49e9-50al y 54c2-6), por citar alguno de los muchos ejemplos, Sócrates retrata la polis ateniense como una instancia que le posibilitó acceder a grandes beneficios, por lo cual, si desobedece las leyes fugándose de prisión, cometería una injusticia contra sus conciudadanos. De hecho, Vlastos (1995) y particularmente Hecht (2011) han identificado el argumento socrático como una formulación del principio de fair play (o fairness).

El principio de fair play, cuyo desarrollo más preciso apareció recién en la segunda mitad del siglo XX, constituye una propuesta para justificar por qué, en materia de obligación política, los ciudadanos de un Estado tienen el deber de obedecer las leyes y sus instituciones. De acuerdo con dicho principio, si un individuo se beneficia del esfuerzo de los participantes de un esquema cooperativo, tiene a su vez el deber de soportar las cargas que establecen los miembros entre sí; de lo contrario, violarían su deber de fair play. Sin embargo, el argumento, aunque sugerente, ha sido objeto de críticas debido a algunas imprecisiones y sobre todo ante la imposibilidad de justificar un deber de obediencia ciudadana cuando, en esquemas complejos como el Estado, los ciudadanos no realizan una formulación expresa sobre la aceptación de los beneficios.

Frente a tal escenario, este trabajo se propone evaluar, a partir de las principales discusiones en la literatura especializada, las virtudes y debilidades del principio de fair play como teoría general de la obligación política. Creemos que muchas de sus objeciones podrían disolverse si consideramos ciertos aspectos, como el valor de la reciprocidad, la carga semántica del concepto de obligación política a la luz del sistema democrático y la participación política. Sin embargo, también debemos reconocer que con esta propuesta se abren nuevos interrogantes, muchos de los cuales muy probablemente exigirán realizar nuevas precisiones que quedarán pendientes para investigaciones mucho más amplias. 
En virtud de los fines propuestos, el trabajo sigue cuatro pasos. En primer lugar, se explicará el principio de fair play y sus principales características de acuerdo con las formulaciones de Hart (1955) y Rawls (1999c). En segundo lugar, veremos la versión de la teoría ofrecida por Klosko (1999, 2004a, 2004b). En tercer término, se analizarán las principales objeciones contra dicha propuesta y, mediante un movimiento que discute su fuerza y validez, se realizarán, en la última parte del trabajo, algunas consideraciones que podrían dar una respuesta a los problemas señalados en la literatura.

\section{LA OBLIGACIÓN POLÍTICA Y EL PRINCIPIO DE FAIR PLAY}

Existe cierto acuerdo entre los especialistas en filosofía política y jurídica de que el concepto de obligación política refiere, en líneas generales, al deber (o la obligación) ${ }^{1}$ moral de los ciudadanos de obedecer las instituciones y las leyes del Estado (Raphael, 1970, p. 94; Singer, 1985, p. 11; Bayón Mohíno, 1991, p. 699). Sin embargo, los acuerdos dentro de la comunidad académica desaparecen tan pronto los especialistas contestan la siguiente pregunta: itodos los integrantes de un Estado o, por lo menos, una buena parte de ellos tienen efectivamente ese requerimiento moral? Las respuestas se dividen grosso modo en dos: mientras que algunos autores mantienen una visión escéptica (Simmons, 1979a; Nozick, 1991; Raz, 1982, 1986), otros aceptan la posibilidad de ofrecer una justificación acerca de por qué los miembros de un Estado tienen el deber de seguir los requerimientos jurídicos (Gilbert, 2006; Horton, 2010; Klosko, 2019). Esta cuestión, para ser más precisos, se conoce en la literatura como "el problema de la obligación política" (Simmons, 1979a, p. 3; Knowles, 2010; Dworkin, 2011, p. 317) y se reduce a la cuestión acerca de si hay razones prima facie o pro tanto por las cuales el cuerpo de ciudadanos debe someterse a la autoridad del derecho y, en tal caso, cuáles serían estas razones.

En la literatura se reconoce que la propuesta teórica que resuelva dicho problema debe satisfacer dos requisitos: en primer lugar, debe cumplir con el requisito de "particularidad", según el cual se exige que la teoría explique por qué los integrantes de un Estado, y solo ellos, deben obedecer el derecho (Simmons, 1979a, pp. 31-34); y, en segundo lugar, la propuesta teórica debe satisfacer el requisito de "generalidad", esto es, justificar un deber u obligación general de obedecer las leyes del Estado y no simplemente la un ciudadano o un grupo minoritario (Simmons, 1979a, pp. 55-56; Klosko, 2004a, p. 3). Entonces, de acuerdo con estos parámetros, cualquier teoría que intente solucionar el problema de la obligación política debe brindar una explicación acerca de por qué los individuos obedecen especialmente su ordenamiento jurídico, así como debe ser lo suficientemente general en su aplicación, es decir, abarcar a la mayoría de los ciudadanos que se hallan bajo la órbita del Estado.

En este trabajo usaremos ambos términos como sinónimos, aunque vale aclarar que en sentido estricto no lo son (Brandt, 1964; Hart, 1977). 
El principio de fair play intenta dar una respuesta a este problema y, ciertamente, parece tener el potencial para hacerlo. Este argumento, que coloca el acento en la estructura de las comunidades como empresas cooperativas constituidas para un fin, tuvo su primera formulación precisa por parte de Hart (1955). Según el profesor de Oxford, cuando varias personas conducen una empresa conjunta estructurada en base a ciertas reglas que restringen su libertad, quienes se han sometido a dichas reglas tienen el derecho a exigir a los individuos que se han beneficiado de tal sumisión que cumplan su parte (Hart, 1955, p. 185). El autor Hart creyó ver aquí, pues, una fuente de derechos y obligaciones que no surgen ni son establecidos a partir de una elección deliberada por parte de aquellos sobre quienes recae la obligación, como sucede por ejemplo cuando alguien formula una promesa o lleva a cabo un acto de consentimiento expreso. En realidad, la dinámica de las relaciones y de la mutua imposición de restricciones establecidas entre los participantes permite la emergencia de dichas obligaciones.

Este tipo de enfoque despertó cierto interés en varios autores, quienes lo vieron como un terreno fructífero para desarrollar algunas ideas en lo que respecta a la lógica de libertades y restricciones al interior de los Estados democráticos. Tal es el caso de Rawls, sin duda uno de los más importantes especialistas en filosofía política de la segunda mitad del siglo XX. El profesor de Harvard abordó el argumento en diversos escritos (Rawls, 1999a y 1999b), pero formuló su versión más precisa en el trabajo Legal Obligation and the Duty of Fair Play de 1964. En un párrafo extenso, pero que conviene reproducir en su integridad, se expone el principio de fair play de la siguiente manera:

Supongamos que existe un esquema de cooperación social justo y mutuamente beneficioso, y que las ventajas que produce solo se pueden obtener si todos, o casi todos, cooperan. Supongamos además que la cooperación requiere un cierto sacrificio por parte de cada persona, o al menos involucra cierta restricción de su libertad. Supongamos finalmente que los beneficios producidos por la cooperación son, hasta cierto punto, gratuitos: es decir, el esquema de cooperación es inestable en el sentido de que si una persona sabe que todos (o casi todos) los demás continuarán haciendo su parte, todavía podrá compartir una ganancia del esquema incluso si no cumple su parte. En estas condiciones, una persona que aceptó los beneficios del esquema está obligada por un deber de fair play a cumplir su parte y a no tomar ventaja de los beneficios gratuitos sin cooperar. (Rawls, 1999c, p. 122)

A la luz de las formulaciones que ofrecen tanto Hart como Rawls se pueden identificar, siguiendo los estudios de Simmons (1979a, pp. 104-108) y Klosko (2004a, p. 34), por lo menos cuatro factores básicos y esenciales sobre los cuales depende el principio de fair play. En primer lugar, debe haber un esquema activo de cooperación social que puede diferir en tamaño, naturaleza y fines. La dinámica de una simple asociación, un programa de ayuda social e incluso la misma sociedad política —muy probablemente la más compleja forma de empresa cooperativa - puede ser pensada de acuerdo con el principio de fair play. Ciertamente, se trata de empresas cooperativas 
o esquemas sociales de cooperación en los que hay libertades y restricciones de distinto tipo y en los que se espera que los integrantes cumplan con las reglas establecidas. Vale aclarar que esto no significa que el principio se aplique a cualquier tipo de organización, sino que hay un elemento material en juego. En efecto, según Rawls (1999c), la empresa cooperativa debe satisfacer dos características: por un lado, debe permitir el beneficio mutuo entre sus integrantes y, por el otro, debe ser justa. El primer aspecto parece ser una condición bastante difícil de negar: de hecho, se puede decir que el concepto mismo de empresa cooperativa comprende la idea de que los participantes realizan ciertos sacrificios para alcanzar algún bien. Sin embargo, no sucede lo mismo con la condición de justicia que exige Rawls. Si este segundo requisito se refiere a la cualidad moral del esquema en sí, no resultaría ser tan esencial, pues existen asociaciones que son claramente injustas y que aun así generan un deber de obediencia entre sus miembros a la luz del principio de fair play: piénsese, por ejemplo, en un grupo de personas dedicadas a cometer delitos. En este sentido, Simmons (1979, p. 114) explica que, los derechos y obligaciones especiales que surgen en virtud del principio se deben a las relaciones de cooperación que existen entre los participantes, pero ello no hace referencia al estatus moral del esquema en sí: el participante beneficiario, pues, debe una parte justa de las cargas simplemente porque otros se han sacrificado para permitirle beneficiarse. Así pues, y aunque no esté exento de problemas (Sartorius, 1999; Simmons, 1979a, pp. 113-117), la condición de Rawls resulta más adecuada si se entiende por ella la distribución equitativa de las cargas y beneficios entre los miembros del esquema cooperativo. Sin ir más lejos, esto condice con la idea básica de justicia que está sobre el principio de fair play: individuos similares deben ser tratados de forma similar (Klosko, 2004a, p. 34).

En segundo lugar, el esquema de cooperación debe involucrar al menos alguna restricción de la libertad personal. Esto supone, como tercer factor, la presencia de ciertas normas que impongan restricciones. No es necesario, por cierto, que estén escritas o definidas de forma expresa, sino que basta con estar establecidas de algún modo que puedan ser comprendidas por los intervinientes del grupo. De hecho, hay muchos esquemas cooperativos en donde las reglas presentan cierta imprecisión y, aun así, funcionan armoniosamente. Lo que importa es que en alguna medida ello debe significar una carga o involucrar cierto sacrificio, aunque sea mínimo, para el participante.

En último término, se requiere la cooperación de la mayoría de los participantes, y no necesariamente de todos, para que la empresa produzca beneficios; de esta manera, al interior de la empresa se puede distinguir entre aquellos que se someten a las reglas y aquellos que no. La teoría del fair play prevé que los beneficios generados en el grupo humano puedan ser obtenidos en algunos casos por alguien que no coopera. Este es precisamente el supuesto del free rider que se da cuando, en una situación de acción colectiva, un individuo que tiene intereses puramente personales y egoístas se beneficia 
de la cooperación de los demás sin llevar a cabo ninguna contraprestación (Roemer, 1994, p. 61). En tal caso, puesto que la base moral del principio es la reciprocidad, quien no hace un sacrificio semejante al que realizaron los demás miembros para sostener la empresa común, actúa sin duda con injusticia (Klosko, 2004a, p. 804).

Hay un quinto requisito que establece Rawls, el cual no encontramos en la formulación de Hart ni tampoco en la versión más reciente de Klosko, la cual veremos con cierto detalle más adelante. En concreto, mientras que estos dos autores dicen que solo es suficiente para la emergencia del deber de fair play la obtención de beneficios de la empresa cooperativa, Rawls, en cambio, va un poco más lejos y exige una aceptación del beneficiario. Específicamente sostiene que la obligación depende de "haber aceptado y de nuestra intención de continuar aceptando los beneficios de un esquema justo de cooperación" (Rawls, 1999c, p. 10). Sin dudas este es un requisito exigente, pues sobre la idea de aceptación subyace un acto de deliberación que se acerca a una expresión de consentimiento. No es lo mismo meramente recibir un beneficio que aceptarlo: uno involucra un acto pasivo, mientras que el otro tiene un carácter activo y, por lo tanto, cuenta con mayor fuerza moral. Tal condición parecería desnaturalizar o, por lo menos, restar valor al principio de fair play como teoría independiente para justificar la obligación de los ciudadanos de obedecer el derecho de su Estado, pues la lleva al terreno de una teoría voluntarista o del consentimiento. En este sentido, pues, cobraría fuerza la sugerencia de Simmons (1979a, pp. 94-95) acerca de que el principio de fair play podría ser una extensión de aquella teoría, ya sea en su versión del consentimiento tácito e incluso expreso. Es discutible, pues, si corresponde incluir la aceptación como un factor esencial de la teoría. Sea como fuere, lo cierto es que, como veremos en la siguiente sección, la exigencia de que haya dicho acto por parte de los beneficiarios es un aspecto importante a los efectos de determinar si, frente a su ausencia, es posible aun hablar de la emergencia de genuinos deberes de obediencia hacia la empresa común de cooperación.

\section{LA VERSIÓN DEL ARGUMENTO DEL FAIR PLAY EN LA TEORÍA DE KLOSKO}

\subsection{El valor del principio en esquemas complejos de cooperación}

En lo que respecta al valor del principio de fair play como teoría que permita justificar la obligación política, el autor más representativo de las últimas décadas es Klosko, quien sigue en esencia los desarrollos de Hart y Rawls. En numerosos trabajos, el profesor de la Universidad de Virginia buscó perfeccionar las formulaciones desarrolladas por aquellos dos pensadores con el fin de sortear una serie de dificultades que fueron remarcadas por varios autores, entre los cuales se destacan Nozick (1991) y Simmons (1979a, 1979b y 2001). En efecto, el argumento del fair play tiene un gran atractivo y en determinadas ocasiones no pareciera tener mayores problemas. Ciertamente, se aplica sin dificultades en esquemas de cooperación que proveen bienes excluibles 
(excludable schemes), esto es, aquellos esquemas en donde los bienes pueden ser percibidos por algunos miembros de la comunidad, pero también negados específicamente a otros (Klosko, 1999, p. 194). Un ejemplo ayudará a explicar este punto. Si un grupo determinado de individuos decide crear un parque de diversiones y sus miembros colocan todas sus fuerzas para concretar dicha empresa, aquellos que disfrutan de los beneficios tienen el deber de compartir las cargas así como el derecho de excluir a aquel sujeto que no haya realizado ningún tipo de colaboración. En el caso de que este se beneficie del proyecto, los demás individuos tienen, entonces, el derecho a exigir que coopere, pues la posibilidad de gozar de los beneficios depende exclusivamente del sacrificio que se haga en el grupo.

Sin embargo, no siempre la aplicación del principio es tan sencilla. Hay muchas dudas acerca de su validez en los esquemas de cooperación que proveen bienes no excluibles (nonexcludable schemes), esto es, bienes que no pueden ser negados a los individuos, como sucede con aquellos de carácter público dentro de un Estado: justicia, orden legal, defensa nacional y seguridad son algunos pocos ejemplos. La razón es clara: en este tipo de esquemas los individuos no tienen la posibilidad de decidir si reciben o no tales beneficios. Este problema, denominado por Klosko (1999, p. 195) como el "argumento limitante" (limiting argument), es un punto que ciertamente desafía el valor de la teoría, pues bajo tales condiciones los beneficiarios no tendrían por qué soportar las cargas del esquema cooperativo (Dagger, 1997, p. 72). De hecho, el propio Rawls (2006) se pronunció en este sentido y expresamente dijo que, de acuerdo con principio de fair play, una persona está obligada a realizar su parte solo si acepta "voluntariamente los beneficios del acuerdo" (p. 113).

Teniendo en mente este problema, también Nozick (1991, pp. 98-99) ha mostrado de manera muy ilustrativa que el principio de fair play permitiría ubicar a las personas bajo una obligación simplemente por haber recibido ciertos beneficios, lo cual es inaceptable. A los fines de explicar este punto, Nozick (1991) pone como ejemplo un grupo de trescientos sesenta y cuatro vecinos que crea un sistema público de entretenimiento, en el cual se le asigna a cada persona del vecindario un día para dirigir el programa. Después de varios meses, llega el turno de un vecino que, si bien no expresó su consentimiento de participar en el programa, se ha beneficiado al abrir ocasionalmente la ventana para escuchar algunos chistes o algo de música. La pregunta que de inmediato surge es si este "espectador inocente" (innocent bystander) está realmente obligado a tomar su turno. La respuesta de Nozick (1991) es que de ningún modo un grupo de personas o una institución puede reclamar obediencia a alguien por el solo hecho de haber sido beneficiado por el esquema cooperativo. No es válido suponer que las personas pueden tener deberes o contraer obligaciones si han obtenido beneficios que no buscaban e incluso hubieran rechazado (Dworkin, 2005, p. 114). Ciertamente, la cuestión clave aquí es que el simple hecho de recibir beneficios no es suficiente para generar una obligación, sino que es necesaria su 
aceptación (Simmons, 1979a, p. 129). Se requiere que el individuo busque obtenerlos y adquirirlos con pleno conocimiento de ello (Simmons, 1979a, p. 131). O, para decirlo con las palabras de Rawls (2006), "los ciudadanos no estarían atados ni siquiera a una constitución, aunque fuese justa, a menos que hubiesen aceptado e intentasen continuar aceptando sus beneficios" (p. 309). Así, pues, la presencia de un elemento volitivo, que sea canalizado de una forma que no deje dudas acerca de la intención del individuo, es determinante en la configuración de la obligación política.

Klosko asumió el desafío de demostrar que el principio de fair play es verdaderamente capaz de generar deberes de obediencia hacia los demás miembros aun en esquemas en donde los bienes no son excluibles u opcionales. En lo que respecta al argumento de Nozick, sostiene que los beneficios que están en juego son tan insignificantes que no podrían generar ninguna obligación; de tal manera, la objeción sería meramente retórica (Klosko, 2004a, p. 39). Sin embargo, Klosko reconoce que, si los bienes en juego tuvieran mayor importancia, entonces la crítica cobraría fuerza. Esto desplaza el centro del debate hacia la cuestión acerca de hasta qué punto emergen deberes cuando los beneficiarios reciben bienes, incluso de gran significancia, aun cuando no tienen la posibilidad de decidir sobre su recepción. La respuesta que ofrece Klosko descansa en la naturaleza de los bienes involucrados. En este sentido, sostiene que la capacidad para generar obligaciones genuinas depende de que satisfagan de forma conjunta tres condiciones: en primer lugar, deben tratarse de "bienes presuntamente beneficiosos" (presumptively beneficial goods), esto es, bienes que "todo hombre supuestamente querría" o, de acuerdo con Rawls (2006), "bienes primarios"; en segundo lugar, los bienes deben valer la pena el esfuerzo de los beneficiaros; y, por último, deben ser distribuidos junto con las cargas de forma equitativa (Klosko, 2004a, pp. 39-57; Klosko, 1999, pp. 197 y 204; Klosko, 2004b, p. 805; Klosko, 2005, p. 6). Comencemos por explicar el primer requisito; de los restantes nos ocuparemos en la próxima sección.

En el caso de los esquemas de cooperación que proporcionan bienes presuntamente beneficiosos, el deber de obediencia se justifica por el hecho de que la importancia de tales beneficios excede o va más allá de la facultad de elección de los participantes. En efecto, tales bienes son indispensables y, en consecuencia, sin ellos no es posible de ninguna manera el desarrollo de la persona. En este sentido, se supone que todos los miembros de la comunidad los querrían con independencia de cuáles serían los detalles de sus planes de vida racionales (Klosko, 2004a, p. 39). Esto sucede, por ejemplo, con la seguridad personal. Muy probablemente, aunque no exista un acto en concreto y específico mediante el cual los ciudadanos hayan aceptado semejante bien público, resulta válido y justificado asumir que todos lo aceptarían; de lo contrario, no existiría condición mínima alguna para garantizar tanto el bienestar particular como el general. 
El argumento es persuasivo y no habría serias razones para rechazarlo. No obstante, Klosko (2004a) advierte que la realidad de los Estados es mucho más compleja y que su descripción de los esquemas que proveen bienes que supuestamente todos desearían es insuficiente. En efecto, en la empresa cooperativa estatal, los ciudadanos no solo se benefician de bienes básicos y fundamentales, como la seguridad, la justicia y el orden legal, sino de otros tipos de bienes: nos referimos a los denominados "bienes discrecionalmente beneficios" (discretionarily beneficial goods) o simplemente "bienes discrecionales", que son todos aquellos que quizá pueden ser deseados por las personas, pero que no son esenciales para el bienestar personal (Klosko, 2004a, p. 85). Entonces, el principio de fair play justificaría que los ciudadanos paguen impuestos con el fin de mantener las fuerzas policiales y toda la maquinaria estatal que garantiza la seguridad ciudadana, pero pierde fuerza al momento de justificar por qué deben hacerlo para que se repare una calle con baches, se corte el césped en las plazas o se poden los árboles.

Al respecto, Klosko (2004a, pp. 86-107) cree encontrar una respuesta al problema extendiendo las funciones de los esquemas cooperativos que proveen bienes presuntamente beneficiosos, de manera tal que se incluyan también los bienes discrecionales. Básicamente, se vale de dos argumentos para poder llevar a cabo este movimiento: un "argumento indirecto" (indirect argument) y otro denominado "argumento institucional" (institutional argument). En lo que respecta al primero, sostiene que cuando un esquema cooperativo provee tanto bienes básicos como bienes discrecionales, en determinadas condiciones ese conjunto de bienes conforma un paquete indivisible de beneficios. La idea detrás de este argumento es que si los bienes presuntamente beneficiosos solo pueden ser provistos junto con un conjunto de bienes discrecionales, entonces se justifica la contribución adicional de los ciudadanos. Así pues, esta última clase de bienes adquiere mayor importancia y hasta son en alguna medida indispensables: no son directamente necesarios para el bienestar individual, pero sí son requeridos indirectamente (Klosko, 2004a, p. 87).

El argumento institucional, por su parte, sostiene que los beneficios derivados del derecho, el orden social y la existencia de un sistema de decisión pública constituyen "bienes institucionales" (institutional goods) que podrían ser recibidos por los individuos si existe una disposición generalizada a cumplir con las cargas de cada uno de acuerdo con el principio de fair play. En palabras de Klosko (2004a), "la esencia del argumento institucional es que, dentro de ciertos límites, el hecho de que los órganos de toma de decisiones del esquema $X$, que suministra a $A$ bienes públicos primarios, establezcan una ley relativa a una política determinada, crea una fuerte obligación para él de cumplir con lo dispuesto" (p. 101). En este sentido, aunque los individuos sean beneficiados con bienes que exceden la órbita de las funciones básicas del Estado, hay un deber de apoyar aun estas políticas, pues de este modo se contribuye a fortalecer la institución (Klosko, 2004a, p. 103). Lo que ataca en última instancia este argumento es el efecto corrosivo de la desobediencia como 
acto que deprecia el valor institucional del Estado de derecho y destruye las condiciones necesarias para el bienestar particular y general.

\subsection{Beneficios y costos equitativos en una distribución justa}

En la propuesta de Klosko, la posibilidad de hablar de un genuino deber de obediencia exige, como hemos mencionado, no solo que los bienes sean presuntamente beneficiosos, sino que además deben valer la pena el esfuerzo de los beneficiaros y ser distribuidos junto con las cargas de forma equitativa. Tomemos para explicar estos dos requisitos un ejemplo de índole ambiental que ofrece el propio Klosko (2004a), aunque con algunas ligeras modificaciones:

Supongamos que existe un país que tiene una severa contaminación en el aire como consecuencia de los gases que generan los automóviles. Hay varias personas con problemas en sus pulmones a las que les cuesta respirar y esta situación se generalizaría a toda la población en un futuro cercano si no se frena la emisión de gases tóxicos. El país, pues, decide establecer una serie de restricciones con respecto a los medios de transporte y dispone que los automóviles deben ser modificados de manera tal que contaminen menos. Todos los ciudadanos cumplen con la normativa, menos $A$, quien no tiene ninguna intensión en realizar su parte. Frente a este escenario se puede decir que $A$ es un free rider, pues se beneficia del esfuerzo de los demás, y que tiene sobre sus hombros un deber genuino de obediencia cuyo cumplimiento puede ser exigido con justicia por el resto del cuerpo ciudadano. (p. 53)

En este caso está claro que el bien en juego vale la pena el esfuerzo de los participantes. La cooperación de todos los individuos se dirige precisamente a frenar la polución y obtener un ambiente sano, equilibrado y apto para el desarrollo humano; de esta manera, resulta legítimo sostener que existe en cabeza de cada uno de los miembros de dicho país un deber de fair play y que la ciudadanía debe cumplir con lo establecido en la normativa. Esta afirmación se encuentra justificada en virtud del bien y los beneficios que están en juego. En efecto, un ambiente que permita una vida saludable constituye un bien que presuntamente todos querrían. Lo mismo podría decirse con respeto a bienes como la seguridad, la justicia y el orden legal: su valor supera sin ninguna duda los costos del esfuerzo que se le exige a cada participante para que el esquema pueda proveerlos. En realidad, los problemas de la teoría emergen cuando se trata de bienes no esenciales, pues no es fácil determinar si su valor justifica esfuerzo alguno. En relación con este punto, como hemos visto, Klosko (2004a, pp. 95-96) sostiene que en muchos casos los bienes forman parte de paquetes indivisibles de beneficios, de manera que su valor puede ser demostrado mediante la vía del argumento indirecto explicado en la sección anterior.

También en el ejemplo ambiental se cumple con el requisito de una distribución equitativa y justa de las cargas y beneficios. Los esfuerzos generados por todos los ciudadanos, si consideramos que todos efectivamente cooperan, son similares: cada 
uno de ellos solo tiene que modificar sus vehículos con el fin de que emitan una menor cantidad de gases tóxicos. Además, los frutos que se obtienen de su trabajo conjunto son distribuidos sin ningún tipo de diferenciación. Todos van a tener un ambiente con menores niveles de contaminación y que les permita llevar a cabo una vida saludable. Se puede afirmar, pues, que la empresa cooperativa ilustrada en este ejemplo supera lo que Klosko (2004a) denomina el "test de distribución justa" (p. 63).

El requisito de distribución justa es una característica definitoria que los esquemas cooperativos deben satisfacer a los efectos de generar el deber de obediencia. La razón que justifica semejante afirmación reside en la idea de justicia que subyace al principio de fair play: el trato igualitario entre los participantes. Entonces, solo bajo la condición de que la distribución de beneficios y cargas sea igualitaria, se puede decir que se satisface el sentido de justicia que informa el fair play; en caso contrario, los individuos no tienen deber alguno de cumplir los requerimientos que se le hagan. El ejemplo del ambiente que hemos utilizado, si bien tiene la virtud de ser bastante ilustrativo en relación con este aspecto, tiene una limitación explicativa vinculada con la complejidad que presentan otros tipos de esquemas cooperativos. En efecto, en la realidad de los Estado, la distribución de bienes y cargas no es tan igualitaria y, además, es una cuestión muy difícil de definir (Klosko, 2004a, p. 54). Ciertamente, algunos individuos deben realizar esfuerzos mucho mayores que otros; incluso en ciertas situaciones la desigualdad está muy marcada. Por ejemplo, en la lucha contra la pandemia generada por la Covid-19, los médicos y enfermeros que están cara a cara con el virus al tratar a los pacientes, tienen una carga mayor que aquellos profesionales de la salud que no tienen contacto directo con los enfermos: ellos arriesgan sus vidas llevando a cabo esta acción y en ocasiones, lamentablemente, la pierden. Sin embargo, de acuerdo con Klosko (2004a), situaciones como estas no cancelan de ningún modo la aplicación del principio de fair play, ya que este no requiere que las cargas de cada uno de los individuos sean idénticas, sino solo que estén asociadas como elementos integrados de un esquema cooperativo. En las diferentes empresas sociales, los individuos tienen roles diferenciados y, en consecuencia, tienen responsabilidades que conllevan distintos esfuerzos; de hecho, esto es algo ínsito a la naturaleza y la dinámica de la mayoría de las asociaciones humanas.

La clave para hablar de una justa distribución radica en la implementación de un proceso de decisión tolerablemente justo mediante el cual se seleccione el mejor sistema posible de reparto de beneficios y cargas (Klosko, 2004a, p. 64). Esto no supone acceder al más perfecto o ideal, sino uno aceptable. A este respecto, los procesos democráticos tienen una clara prioridad: garantizan la participación ciudadana, pues todas las voces pueden ser escuchadas e, incluso en situaciones en las cuales hay fuertes desacuerdos, existen varios mecanismos que permiten canalizar los disensos ciudadanos como una presentación formal ante la administración de justicia, una protesta pacífica o incluso la desobediencia civil (Klosko, 2004a, p. 65). 
Vale aclarar que el énfasis en los procesos de deliberación no está puesto solo en un sentido de justicia formal o procedimental, sino que ellos comprenden un aspecto sustantivo, ya que la elección de un criterio de distribución debe apoyarse en la argumentación racional, someterse al control cívico y ser congruente con una concepción de justicia ampliamente reconocida por los participantes. Se trata, pues, más que un estándar de "equidad perfecta" (perfect fairness), de "equidad tolerable" (toreable fairness) que expresa con mayor verdad y sensatez la realidad imperfecta de las empresas cooperativa y en especial la del Estado (Klosko 2004a, pp. 66-67). Entonces, de acuerdo con el sistema democrático, en los casos en que ciertos individuos estén en desacuerdo con el estándar de distribución adoptado por la mayoría, deben estar dispuestos a aceptarlo siempre y cuando la decisión caiga dentro de los límites de una equidad tolerable. Esto constituye el cumplimiento de un principio que Klosko (2004a, p. 72) denomina "regla de prioridad" (precedence rule).

Por lo demás, el autor insiste en que el requisito de justa distribución debe ser pensado con respecto al esquema como un todo en lugar de colocarse el acento en uno o algunos pocos individuos en particular. Esta es una condición mucho más fuerte sobre la cualidad moral de los esquemas en sí mismos que, sin embargo, no descuida la situación singular de los participantes: no es de ningún modo una visión totalitaria donde el todo le puede exigir a la parte que coopere aun en su propio perjuicio. Al contrario, Klosko (2004a, p. 64) sostiene que el requisito de distribución justa no se satisface con respecto a los individuos concretos a menos que el esquema cooperativo como un todo sea razonablemente justo. En este sentido, en los esquemas manifiestamente injustos las obligaciones de cooperación se disuelven: como los deberes de los participantes tienen un carácter prima facie, son anulados por la injusticia del esquema. Lo que se entiende por esquemas injustos es algo que no se puede definir de antemano mediante reglas abstractas, sino que en cada caso se tiene que evaluar si se va más allá de los contornos de lo tolerable (Klosko, 2004a, p. 122). La teoría del fair play, si bien en este aspecto es imprecisa — como no podía ser de otro modo dado la complejidad de la vida real—, es capaz no solo de fundamentar la obligación política, sino de establecer límites al cumplimiento de los deberes, determinados por la justicia del esquema (Klosko, 2004a, p. 125).

\section{EL VALOR DE LA TEORÍA DEL FAIR PLAY BAJO EXAMEN}

\subsection{Las principales debilidades del principio}

El principio de fair play formulado por Hart y Rawls, y explotado ingeniosamente por Klosko, constituye una propuesta prometedora en lo que se refiere al problema de la obligación política. Sin embargo, no deja de tener puntos débiles al momento de justificar por qué todos (o la mayoría) de los ciudadanos de un Estado en particular deben someterse a la autoridad del derecho. En líneas generales, los principales 
inconvenientes de la teoría pueden resumirse en cuatro, muchos de los cuales tienen una respuesta en la versión de Klosko que, sin embargo, no termina de convencer en modo alguno a los críticos. En las seccione previas se hizo mención de algunos de estos problemas, pero por cuestiones de orden y claridad expositiva conviene sintetizarlos muy rápidamente. Esto nos permitirá delinear el terreno para poder, en un doble movimiento de crítica y superación, ofrecer algunos puntos novedosos que podrían contribuir a mejorar la teoría.

En primer lugar, el principio no puede justificar por qué, en el marco de esquemas de cooperación de bienes no excluibles, los individuos deben obediencia cuando los bienes en juego son discrecionales. La respuesta de Klosko es que este tipo bienes permite la provisión de los bienes primarios y que la relación entre ambas categoríases tan estrecha que conforman un paquete indivisible que justifica las contribuciones adicionales. Entonces, siempre y cuando los bienes sirvan de modo mediato o inmediato en la satisfacción de las necesidades humanas, habrá en cabeza de los ciudadanos un deber de fair play de someterse al derecho. Sin embargo, el problema de este planteo es que el concepto de "paquete indivisible" es muy impreciso y en muchos casos no es fácil demostrar cómo existe una conexión entre los bienes discrecionales y los bienes primarios. No está claro, por ejemplo, por qué pagar para que se corte el césped de las plazas contribuye a la seguridad personal o la defensa de la nación. Así pues, es una pobre justificación igualar, como parece hacer Klosko, la obligación debida por la percepción de bienes esenciales con nuevas obligaciones que el Estado imponga o vaya imponiendo para respaldar la provisión de bienes discrecionales. Sin violentar la autonomía de la voluntad, no se pueden establecer obligaciones a alguien cuando no las ha contraído ni tampoco se puede presumir su existencia frente a la ausencia de una aceptación voluntaria. En caso contrario, la imposición de semejantes cargas mediante la provisión de un paquete indivisible de bienes sería una suerte de tiranía (Simmons, 1987, pp. 274-275). Sin duda, pues, la cuestión señalada por Rawls (2006, p. 113) acerca de que los ciudadanos no están obligados a cumplir las reglas de un esquema justo a menos que hubieren aceptado sus beneficios y mantengan la decisión de seguir aceptándolos, sigue desafiando el valor de la teoría del fair play.

En segundo lugar, el argumento tiene una ambigüedad conceptual. En efecto, resulta difícil establecer cuándo un individuo dentro de una organización política se beneficia (Dworkin, 2005, p. 144). Las respuestas podrían ser dos. En primer lugar, se puede decir que una persona obtiene beneficios cuando su situación general en el Estado es mejor que aquella que tendría en otras circunstancias. Esto, sin embargo, exige realizar una comparación entre la situación de la persona en dicho esquema y su situación en otro punto de referencia, lo cual conduce a un callejón sin salida. La segunda posibilidad sería decir que la idea de beneficio no significa un mejoramiento de su bienestar, sino sencillamente que ha recibido lo que le 
corresponde de acuerdo con las normas de la organización sin discriminación alguna. Esta respuesta también es problemática, pues no se ve cómo ello pueda, en palabras de Dworkin (2005), "dar una razón positiva de por qué [un individuo] debe aceptar sus leyes como obligaciones" (p. 144). En efecto, que "haya sido tratado con justicia por su comunidad de acuerdo con sus propias normas no le garantiza ninguna otra ventaja material" (Dworkin, 2005, p. 145).

En tercer término, tampoco está del todo claro el grado de cooperación que deben realizar los individuos para exigir obediencia. Si una persona, sea por lo que fuere, no coopera al mismo nivel que los demás, cabe preguntarse hasta qué punto tiene el derecho de requerir a los demás miembros de la empresa cooperativa que hagan su parte como deberían hacerlo. Los esquemas cooperativos no son homogéneos en lo que respecta al grado de cooperación que llevan a cabo los participantes, tal como sucede en el Estado. Al contrario, hay quienes tienen más cargas e incluso menos beneficios en virtud de su posición social (accidental o voluntariamente asumida), de manera que el requerimiento de cooperación va a ser diferente, y eso constituye una situación de desigualdad (Smith, 1999, p. 82).

Por último, el principio por sí solo no logra justificar por qué a un individuo se le debe exigir obediencia cuando su sumisión a las reglas no produce ningún beneficio o su desobediencia no daña directa o indirectamente los intereses del que cumple su parte (Smith, 1999, pp. 80-81). Esto quiere decir que si un sujeto se beneficia de la sumisión de los demás, no parece justo decir que estos tienen el derecho de reclamar obediencia en el caso de que sus intereses no hayan sido afectados o no tuviesen perjuicio alguno, salvo que la cuestión solo pase por cumplir con la promesa. Supongamos que en el ejemplo ofrecido en la sección anterior acerca de la reducción de la contaminación ambiental, el uso que le da un ciudadano a su automotor no incrementa en modo alguno los niveles de gases tóxicos en el aire. En esta situación, si decide no modificar el vehículo de acuerdo con la normativa establecida, incumple con su parte, pero lo cierto es que aun así no genera perjuicio alguno sobre los demás miembros. Se podría decir, de todas formas, que es la falta de cooperación en sí misma lo que daña el esquema cooperativo, más allá de sus efectos. No obstante, esta respuesta tiene un alcance limitado. Existen situaciones en que las que la desobediencia, antes que la sumisión a las reglas, es la que puede a veces generar más beneficios. Siguiendo con el ejemplo ambiental, supongamos que todos los ciudadanos han acordado, según lo dispuesto en la normativa, solo modificar el sistema de combustión de sus autos de forma tal que consuman menos combustible y generen, pues, una menor emisión de gases tóxicos. Supongamos que un ciudadano decide no cumplir con su parte y directamente compra un auto eléctrico para contribuir aún más en la reducción de la contaminación del aire, algo que no estaba previsto en el acuerdo ni en la normativa. En este caso, el incumplimiento de lo acordado es lo que precisamente produce mayores 
beneficios, de manera que carecería de sentido decir que ello genera en sí mismo un perjuicio hacia la comunidad o, por lo menos, es contraintuitivo.

\subsection{Una defensa de la teoría}

Los tres últimos problemas brevemente desarrollados no tienen la entidad suficiente como para conmover el principio de fair play ni mucho menos para descartarlo como propuesta válida en lo que respecta al problema de la obligación política. En efecto, la fuerza de las objeciones se desvanece tan pronto se considera el valor moral sobre el cual se sustenta dicho principio: la reciprocidad en las relaciones humanas. Esta es, de acuerdo con Becker (1986, p. 3), una virtud según la cual los hombres están obligados, por una cuestión moral, a devolver en la misma proporción los bienes recibidos por el esfuerzo de otros y a reparar los daños que han ocasionado. En casi la mayor parte de las instituciones sociales (como, por ejemplo, la amistad), económicas (el mercado), jurídicas (una sociedad civil) o incluso políticas (el Estado), la reciprocidad juega, de algún modo u otro, un rol central. De hecho, no resulta exagerado afirmar que en las relaciones intersubjetivas su ausencia es algo un tanto extraño. No se puede negar, sin embargo, que muchas veces dentro de aquellos tipos de relaciones humanas hay involucradas otras cuestiones, como intereses personales y hasta egoístas que empañan el desarrollo del principio de fair play; pero esto no significa que no haya, de acuerdo con la naturaleza de la institución, una pretensión de correspondencia mutua, un trato recíproco o intercambio igualitario entre los intervinientes. Tales factores son propios de la dinámica misma de la relación y hasta en ocasiones son necesarios para preservar su integridad.

El desconocimiento del valor de la reciprocidad constituye negar los cimientos mismos sobre los cuales se asientan las relaciones entre los seres humanos. Cuando los individuos se reúnen y relacionan bajo determinada forma, la asociación no constituye un simple agregado de individuos que agota su sentido en la satisfacción de los intereses particulares. Esta es una mirada muy pobre de lo que involucra la vida intersubjetiva y la configuración de distintos esquemas, asociaciones o grupos humanos. Al contrario, supone algo más, tanto ética como ontológicamente. En el caso del Estado, que es la organización que por supuesto más nos interesa en este trabajo, se trata de una empresa cooperativa muy compleja, cuyo valor no se reduce a obtener meramente beneficios particulares. En realidad, es una institución que en sí misma tiene dignidad propia y amerita ser conservada en el tiempo: es una instancia distinta a sus bases que establece las condiciones de posibilidad para que sus miembros puedan realizar su plan de vida y, en última instancia, la comunidad en su integridad alcance el bienestar general.

No es posible decir lo mismo con respecto al primer inconveniente señalado, que es aquel que precisamente autores como Nozick (1991) destacan mediante el "argumento limitante", el cual hemos explicado brevemente en la sección 2.1. El valor 
de la reciprocidad no alcanza para sortear ese obstáculo ni el papel que cumple el Estado como organización digna de ser conservada en el tiempo. Sin embargo, dicha objeción se diluye si se adopta un camino ligeramente distinto al de Klosko, pero que conserva un mismo espíritu. El camino involucra, por lo menos, dos pasos: uno conceptual y otro procedimental.

En primer lugar, resulta necesario reivindicar la densidad semántica del concepto de obligación política. En efecto, el concepto tiene una doble valencia que involucra tanto la sumisión a la autoridad del derecho como la participación en la construcción de las normas que regulan la vida social. En la literatura, por lo general, suele ser pensado en su dimensión pasiva como una simple exigencia de someterse a las leyes e instituciones del Estado. Pero en realidad es mucho más rico semánticamente. En efecto, el individuo desarrolla su plan de vida en el Estado, convive y se entreteje con sus diversas instituciones sociales, jurídicas y políticas. En este proceso, mantiene una relación especial con la organización estatal (Johnson, 1974, pp. 533-535; Singer, 1985, p. 13); un vínculo complejo que comprende las razones morales por las cuales se justifica el cumplimiento de sus disposiciones jurídicas y el deber de apoyar las instituciones (Horton, 2010, p. 11-13). Así pues, el concepto de obligación política es sumamente particular, al punto tal que autores como Polin (1971, p. 35) dicen que se trata de una obligación sui generis.

En el caso puntual de los Estados democráticos, la relación ciudadano/comunidad política no solo significa cumplir con las cargas que se le imponen a los individuos y quedar ajeno a todo lo demás que sucede en la vida institucional. Al contrario, comprende además el deber de participar en la configuración del esquema político, de intervenir directa o indirectamente. En este sentido, Johnson (1975, p. 26) explica que el concepto de obligación política es un concepto positivo que requiere más del miembro del Estado que de una pasiva aquiescencia en la determinación de sus deberes. Exige, pues, la intervención ciudadana activa y responsable.

Que el concepto de obligación política no se trata de una mera sumisión a la ley, lo muestra que el ciudadano, además de tener simples cargas, tiene responsabilidades muy importantes que constituyen acciones positivas destinadas precisamente a la configuración del Estado, el fortalecimiento de sus instituciones y su legitimidad. Así, por ejemplo, esto sucede con el deber de votar, de defender la nación en tiempos de crisis e incluso de ejercer la resistencia ante la emergencia de un gobierno ilegítimo. En estos casos, los ciudadanos no tienen una simple exigencia de cumplir con la obligación como si se tratara de la norma jurídica que prohíbe cruzar con el vehículo un semáforo en rojo. Al contrario, son instancias que involucran una intervención política fuerte y que dan cuenta del sentido más profundo que recoge el concepto de obligación política. Un Estado democrático en el que el conjunto de sus ciudadanos fuesen meros sujetos pasivos no sería más que como el David de Miguel Ángel, una escultura muy hermosa, pero inerte y sin vida. La democracia supone obediencia al 
derecho, pero también asumir el compromiso de estar informado y ejercer la constante tarea de mantener y fortalecer las instituciones mediante la participación. Sin duda, ser un agente activo en la configuración de la empresa estatal resulta clave para que el sentido del cuerpo político no se agote en una mera agrupación o suma de individualidades, sino en una instancia ética y ontológicamente más elevada.

En este orden de ideas, la objeción introducida mediante el argumento limitante sería válida solo en un aspecto: siempre y cuando la obligación política sea considerada meramente como algo pasivo. Sin embargo, en lo que respecta a la faz activa del concepto, el argumento no tiene efecto alguno. Ahora bien, este paso, si bien es necesario para dar una respuesta aceptable al problema, no lleva a ningún lado si no se da un segundo paso. En efecto, debe haber un mecanismo legítimo y justo de decisión acerca de qué bienes no esenciales los ciudadanos estarían dispuestos a recibir y aprovechar. Esta cuestión, que es naturalmente de orden procedimental y ha sido sugerida por Moauro (2011), puede materializarse de distintas formas, como en audiencias, procesos especiales u otros medios. Lo importante es que exista un mecanismo de índole democrático a través del cual, mediata o inmediatamente, los ciudadanos puedan hacer escuchar su voz sobre los bienes discrecionales por los cuales estarían dispuestos a realizar contribuciones adicionales. No desconocemos que el planteo es ambicioso y que en muchos casos es muy difícil ponerlo en práctica debido a la complejidad de los procesos de toma de decisiones públicas. No obstante, se debe reconocer que, en los tiempos actuales, los medios de comunicación y las vías por las cuales los ciudadanos pueden expresarse en el espacio político son cada vez más sencillos y eficaces, de manera que aquel problema no debería ser un obstáculo. Si se aceptan tales ideas, entonces el sistema justo de decisión debería ser un cuarto requisito que se podría agregar a los tres ya establecidos por Klosko (2004a), el cual justifica puntualmente por qué los ciudadanos deben realizar contribuciones adicionales por los bienes discrecionales recibidos.

Hay una característica más que Klosko (2004a, pp. 101-107) desarrolla en el marco del argumento institucional, pero que debe ser elevada al rango de requisito de la teoría. El principio de fair play funciona siempre y cuando haya cierto nivel de obediencia. Cuando todos o la mayor parte de los participantes desobedecen las reglas, no hay posibilidad de realizar exigencia alguna. La empresa común debe tener un compromiso robusto, ya que el incumplimiento a sus normas ataca directamente la base sobre la que se asienta el principio, esto es, la reciprocidad. Al mismo tiempo, esto genera una depreciación del valor de la ley que repercute en la posibilidad de promover el bien común (Tale, 2004). Entonces, en tales circunstancias las expectativas de que los miembros hagan su parte se verían por completo paralizadas y, lo que es peor aún, se pondría en riesgo la integridad de la asociación. Ningún hombre racional realizaría sacrificio alguno allí donde la regla es la desobediencia y el esquema no puede garantizar, debido al caos y la anomia, que los esfuerzos no sean en vano. 
A la luz de estas consideraciones, pues, se puede decir que desde la óptica de la teoría del fair play la obligación de obedecer las disposiciones del Estado se encuentra justificada si se satisfacen de forma conjunta cinco condiciones: en primer lugar, debe tratarse del reparto de "bienes presuntamente beneficiosos" o que "todo hombre supuestamente querría" debido a su carácter primario; en segundo lugar, los bienes deben valer la pena el esfuerzo de los beneficiaros; en tercer término, deben ser distribuidos junto con las cargas de forma equitativa; como cuarto elemento, los bienes discrecionales u opcionales deben ser establecidos entre los participantes del esquema cooperativo mediante un mecanismo justo de decisión que permita la materialización de la obligación política en su faz activa; y, finalmente, debe haber cierto grado de obediencia efectiva capaz de mantener el valor de la reciprocidad y conservar la integridad de la empresa común.

\section{CONCLUSIONES}

En el marco de un Estado democrático, la teoría del fair play tiene un gran potencial para justificar el deber de los ciudadanos de obedecer el derecho. Ciertamente, el principio reflejaría una cuestión moral subyacente al desarrollo de las relaciones intersubjetivas, la adquisición de beneficios y la realización de sacrificios que se dan en el seno de un conjunto de individuos que se ven a sí mismos como miembros de una empresa cooperativa común orientada a la realización de determinados fines. En este sentido, McDermott (2004), si bien es un crítico de la teoría, reconoció que "la gran fuerza de la teoría del fair play es que se basa en una afirmación empírica indiscutible: en muchas sociedades las personas realmente se benefician de la provisión de bienes por parte de sus comunidades políticas" (p. 217). Sin embargo, frente a este dato fáctico, la pregunta que aún subsiste es si verdaderamente existe algo así como un deber de fair play en cabeza de todos (o la gran mayoría) de los participantes del Estado.

Muchos autores consideran que el principio constituye una propuesta poco convincente e incluso irrelevante como teoría general de la obligación política. Hemos tratado de demostrar, no obstante, que mediante algunos requisitos complementarios a la versión de Klosko, se puede ofrecer una respuesta sólida a sus objeciones. En caso de que las dudas todavía persistan, quizá el camino más prudente sea complementarla con otras propuestas, como por ejemplo las teorías asociativas o las voluntaristas. De hecho, el propio Klosko (2005, pp. 98-121, 2019), en sus últimos trabajos sobre el tema, adoptó un enfoque en el que combina el principio de fair play con el deber natural de ayuda mutua y el principio del bien común. Pero antes de llegar a esa instancia, lo cual implica en cierto modo abandonar la pretensión de ofrecer una justificación teórica lo más sencilla posible, creemos que la teoría del fair play por sí misma tiene todavía mucho que decir. 


\section{REFERENCIAS}

Bayón Mohíno, J. C. (1991). La normatividad del derecho: deber jurídico y razones para la acción. Centro de Estudios Constitucionales.

Becker, L. (1986). Reciprocity. Routledge.

Brandt, R. B. (1964). The Concepts of Obligation and Duty. Mind, 73(291), 374-393.

Dagger, R. (1997). Civic Virtues: Rights, Citizenship, and Republican Liberalism. Oxford University Press.

Dworkin, R. (2005). El imperio de la justicia. Gedisa.

Dworkin, R. (2011). Justice for Hedgehogs. Harvard University Press.

Gilbert, M. (2006). A Theory of Political Obligation: Membership, Commitment, and the Bonds of Society. Oxford University Press.

Hart, H. L. A. (1955). Are There Any Natural Rights? Philosophical Review, 64(2), 175-191.

Hart, H. L. A. (1977). Obligación jurídica y obligación moral, Cuadernos de Crítica, 3, 5-33.

Hecht (2011). Fair Play: Resolving the Crito-Apology Problem, History of Political Thought, 32(4), 543-564.

Horton, J. P. (2010). Political Obligation. Pelgrave Macmillan.

Johnson, K. (1974). Perspectives on Political Obligation: A Critique and a Proposal, The Western Political Quarterly, 27(3), 520-535.

Johnson, K. (1975). Political Obligation and the Voluntary Association Model of the State, Ethics, 86(1), 17-29.

Klosko, G. (1999). Presumptive Benefit, Fairness, and Political Obligation. En W. A. Edmundson (Ed.), The Duty to Obey the Law (pp. 193-212). Rowman \& Littlefield.

Klosko, G. (2004a). The Principle of Fairness and Political Obligation. Rowman \& Littlefield.

Klosko, G. (2004b). Multiple Principles of Political Obligation, Political Theory, 32(6), 801-824.

Klosko, G. (2005). Political Obligations. University Press.

Klosko, G. (2019). Why Should We Obey the Law? Polity Press.

Knowles, D. (2010). Political Obligation. A critical introduction. Routledge.

McDermott, D. (2004). Fair-Play Obligations, Political Studies, 52, 216-232. https://onlinelibrary.wiley. com/doi/abs/10.1111/j.1467-9248.2004.00476.x.

Moauro, L. (2011). A Critical Assessment of George Klosko's Version of the Principle of Fair Play, Stance, 4, 61-68. https://openjournals.bsu.edu/stance/article/view/1785.

Nozick, R. (1991). Anarquía, Estado y Utopía. F.C.E.

Platón (2010). Critón. Traducción de C. Eggers Lan. Eudeba.

Polin, R. (1971). L'obligation politique. Presses Universitaires de France.

Raphael, D. D. (1970). Problems of Political Philosophy. Praeger Publishers.

Rawls, J. (1999a). Justice as Fairness. En S. Freeman (Ed.), Collected Papers (pp. 47-72). Harvard University Press. 
Rawls, J. (1999b). The Sense of Justice. En S. Freeman (Ed.), Collected Papers (pp. 96-116). Harvard University Press.

Rawls, J. (1999c). Legal Obligation and the Duty of Fair Play. En S. Freeman (Ed.), Collected Papers (pp. 117-129). Harvard University Press.

Rawls, J. (2006). Teoría de la Justicia. FCE.

Raz, J. (1982). La Autoridad del Derecho. Ensayos sobre Moral y Derecho. UNAM.

Raz, J. (1986). The Morality of Freedom. Clarendon Press.

Roemer, A. (1994). Introducción al análisis económico del derecho. FCE.

Sartorius, R. (1999). Political Authority and Political Obligation. En W. A. Edmundson (Ed.), The Duty to Obey the Law (pp. 143-157). Rowman \& Littlefield.

Simmons, J. A. (1979a). Moral Principles and Political Obligations. University Press.

Simmons, J. A. (1979b). The Principle of Fair Play, Philosophy \& Public Affairs, 8(4), 307-337.

Simmons, J. A. (1987). The Anarchist Position: A Reply to Klosko and Senor, Philosophy \& Public Affairs, 16(3), 269-279.

Simmons, J. A. (2001). "Fair Play and Political Obligation: Twenty Years Later". En Justification and Legitimacy: Essays on Rights and Obligations (pp. 27-42). University Press.

Singer, P. (1985). Democracia y desobediencia. Ariel.

Smith, M. B. E. (1999). Is There a Prima Facie Obligation to Obey the Law? En W. A. Edmundson (Ed.), The Duty to Obey the Law (pp. 193-212). Rowman \& Littlefield.

Tale, C. (2004). El valor de la ley positiva, Opinión jurídica, 3(5), 87-102. https://revistas.udem.edu.co/ index.php/opinion/article/view/1326/1316

Vlastos, G. (1995). Socrates and Political Obedience and Disobedience. En D. W. Graham (Ed.), Gregory Vlastos. Studies in Greek Philosophy. Socrates, Plato, and Their Tradition, vol. 2 (pp. 30-42). Princeton University Press. 